\title{
THE EXPLICIT FORMULAS FOR REPRODUCING KERNEL OF SOME HILBERT SPACES
}

\author{
E. MORADI, E. BABOLIAN, AND S. JAVADI
}

Received 06 December, 2014

\begin{abstract}
This paper is devoted to finding explicit formulas for two kinds of reproducing kernels in some reproducing kernel spaces. Usually for calculating the reproducing kernel of space $W_{2}^{m}[a, b]$, one must solve a large system of ordinary differential equations with $4 m$ equations in $4 m$ unknowns. Hence, it is worthy to have explicit formulas to save time and computational efforts. The advantage of using these formulas is clearly seen in the plotted graphs.
\end{abstract}

2010 Mathematics Subject Classification: 46E22; 65L05

Keywords: system of ordinary differential equations, reproducing kernel, explicit formula

\section{INTRODUCTION}

In recent decades, the reproducing kernel Hilbert space method has been considered by many authors. Reproducing kernel Hilbert space method is an effective method for solving many problems, including ordinary, partial, integro-differential, integral equations, etc. [1-25].

To obtain reproducing kernel we have to perform complex calculations at high volumes. So we decided to introduce, in this paper, explicit formulas to calculate unknown coefficients of the reproducing kernel. That will save time and reduce the high cost of computing. First, we divide reproducing kernel spaces in terms of the types of problems, different initial and boundary conditions, the inner product, and the necessary norm on the environment. The first class (i.e. $\left.W_{2}^{m}[a, b]\right)$ is intended for problems without initial and boundary conditions and the second class (i.e. $\left.W_{2, a}^{n+1}[a, b]\right)$ is devoted to the problems with the initial conditions. The findings of this article for readers, especially for users of reproducing kernel method, is helpful in time saving.

The structure of this paper is as follows. In Section 2 we introduce some reproducing kernel spaces. We devote Section 3 to finding explicit formulas for reproducing kernels. We end the paper with conclusions. 


\section{REPRODUCING KERNEL HILBERT SPACES}

In this section, we first define the reproducing kernel and reproducing kernel space, and then we introduce some reproducing kernel spaces.

Definition $1([1,4])$. Let

$$
\mathscr{H}=\{f \mid f \text { is a real valued function on } \mathcal{X}, \mathcal{X} \text { is an abstract set }\}
$$

be a Hilbert space, with inner product

$$
\langle f(.), g(.)\rangle_{\mathscr{H}}, \quad f, g \in \mathscr{H} .
$$

If there exists a function $K(x,.) \in \mathscr{H}$ for each fixed $x \in \mathcal{X}$ and for any $f \in \mathscr{H}$

$$
\langle f(.), K(x, .)\rangle_{\mathscr{H}}=f(x),
$$

then $K(x,$.$) is called the reproducing kernel of \mathscr{H}$ and Hilbert space $\mathscr{H}$ is called the reproducing kernel space.

For solving some functional equations such as integral and differential equations using the reproducing kernel Hilbert space method, we use the reproducing kernel space $W_{2}^{m}[a, b]$ defined as follows.

Definition 2 ([4]). For $m \in \mathbb{N}$,

$W_{2}^{m}[a, b]=\left\{u \mid u^{(m-1)}\right.$ is an absolutely continuous function, $\left.u^{(m)} \in L^{2}[a, b]\right\}$.

The inner product and the norm in the function space $W_{2}^{m}[a, b]$ are defined as

$$
\begin{gathered}
\langle u, v\rangle_{m}=\sum_{i=0}^{m-1} u^{(i)}(a) v^{(i)}(a)+\int_{a}^{b} u^{(m)}(\tau) v^{(m)}(\tau) d \tau, \quad u, v \in W_{2}^{m}[a, b] \\
\|u\|_{m}=\sqrt{\langle u, u\rangle_{m}}, \quad u \in W_{2}^{m}[a, b] .
\end{gathered}
$$

To solve initial value problems including ordinary differential equations, integrodifferential equations and differential equations of fractional order, we define the reproducing kernel space $W_{2, a}^{n+1}[a, b]$ as follows.

Definition 3. For $n \in \mathbb{N}$,

$$
W_{2, a}^{n+1}[a, b]=\left\{u \in W_{2}^{n+1}[a, b] \mid u^{(j)}(a)=0, j=0, \ldots, n-1\right\} .
$$

The inner product and the norm in the function space $W_{2, a}^{n+1}[a, b]$ are defined as

$$
\begin{gathered}
\langle u, v\rangle_{n, a}=u^{(n)}(a) v^{(n)}(a)+\int_{a}^{b} u^{(n+1)}(\tau) v^{(n+1)}(\tau) d \tau, \quad u, v \in W_{2, a}^{n+1}[a, b] \\
\|u\|_{n, a}=\sqrt{\langle u, u\rangle_{n, a}}, \quad u \in W_{2, a}^{n+1}[a, b] .
\end{gathered}
$$




\section{EXPLICIT FORMULAS FOR REPRODUCING KERNELS}

In this section we investigate explicit formula for each one of the reproducing kernel Hilbert spaces introduced in Definitions 2 and 3.

\subsection{Reproducing kernel of the space $W_{2}^{m}[a, b]$}

Assume that function $K^{\{m\}}(x, t) \in W_{2}^{m}[a, b]$ satisfies the following generalized differential equation

$$
\left\{\begin{array}{l}
(-1)^{m} \frac{\partial^{2 m} K^{\{m\}}(x, t)}{\partial t^{2 m}}=\delta(t-x), \\
\frac{\partial^{j} K^{\{m\}}(x, t)}{\partial=a}=\left.(-1)^{m-j-1} \frac{\partial^{2 m-j-1} K^{\{m\}}(x, t)}{\partial t^{2 m-j-1}}\right|_{t=a}, \\
\left.\frac{\partial^{m+j} K^{j} K^{j m\}}(x, t)}{\partial t^{m+j}}\right|_{t=b}=0,0 \leq j<m .
\end{array}\right.
$$

where $\delta$ is the Dirac delta function. The following theorem holds.

Theorem 1 ([4]). Under the assumptions of Eq. (3.1), Hilbert space $W_{2}^{m}[a, b]$ is a reproducing kernel Hilbert space with the reproducing kernel function $K^{\{m\}}(x,$.$) ,$ namely for each $u \in W_{2}^{m}[a, b]$ and any fixed $x \in[a, b]$, it follows that

$$
\left\langle u(.), K^{\{m\}}(x, .)\right\rangle_{m}=u(x) .
$$

While $x \neq t$, function $K^{\{m\}}(x, t)$ is the solution of the following linear homogeneous differential equation of order $2 m$,

$$
(-1)^{m} \frac{\partial^{2 m} K^{\{m\}}(x, t)}{\partial t^{2 m}}=0,
$$

with the boundary conditions:

$$
\left\{\begin{array}{l}
\left.\frac{\partial^{j} K^{\{m\}}(x, t)}{\partial t^{j}}\right|_{t=a}=\left.(-1)^{m-j-1} \frac{\partial^{2 m-j-1} K^{\{m\}}(x, t)}{\partial t^{2 m-j-1}}\right|_{t=a}, \\
\left.\frac{\partial^{m+{ }^{\prime}} K^{\{m\}}(x, t)}{\partial t^{m+j}}\right|_{t=b}=0,0 \leq j<m .
\end{array}\right.
$$

We know that Eq. (3.2) has characteristic equation $\lambda^{2 m}=0$, and the eigenvalue $\lambda=0$ is a root with multiplicity $2 m$. Hence, the general solution of the system (3.1) is

$$
K^{\{m\}}(x, t)= \begin{cases}\sum_{i=1}^{2 m} c_{i}(x)(t-a)^{i-1}, & t \leq x, \\ \sum_{i=1}^{2 m} d_{i}(x)(t-a)^{i-1}, & t>x .\end{cases}
$$

Now, we are ready to calculate the coefficients $c_{i}(x)$ and $d_{i}(x), i=1, \ldots, 2 m$. Since

$$
(-1)^{m} \frac{\partial^{2 m} K^{\{m\}}(x, t)}{\partial t^{2 m}}=\delta(t-x)
$$

we have

$$
\begin{cases}\left.\frac{\partial^{j} K^{\{m\}}(x, t)}{\partial t^{j}}\right|_{t=x^{-}} ^{t=x^{+}}=0, & j=0, \ldots, 2 m-2, \\ \left.\frac{\partial^{j} K^{\{m\}}(x, t)}{\partial t^{j}}\right|_{t=x^{-}} ^{t=x^{+}}=(-1)^{m}, & j=2 m-1 .\end{cases}
$$


Therefore, using Eqs. (3.1) and (3.5), we have a system of $4 m$ equations with $4 m$ unknowns $\left(c_{i}(x)\right.$ and $\left.d_{i}(x), i=1, \ldots, 2 m\right)$. By solving this system the unknown coefficients of Eq. (3.4) are uniquely determined. Solving the above system needs high CPU time.

We have obtained the following formula for calculating the reproducing kernel of $W_{2}^{m}[a, b]$.

Theorem 2. Let

$$
K^{\{m\}}(x, t)= \begin{cases}R^{\{m\}}(x, t), & t \leq x, \\ L^{\{m\}}(x, t), & t>x,\end{cases}
$$

be the reproducing kernel function of the reproducing kernel Hilbert space $W_{2}^{m}[a, b]$. Then, $R^{\{m\}}(x, t)$ is given by the following formula

$$
R^{\{m\}}(x, t)=\sum_{i=0}^{m-1} \sum_{j=0}^{1}\left((-1)^{i j} \frac{(x-a)^{i+(m-2 i-1) j}(t-a)^{m j+i}}{(i+(m-2 i-1) j) !(m j+i) !}\right),
$$

and $L^{\{m\}}(x, t)=R^{\{m\}}(t, x)$.

Proof of Theorem 2. It is enough to prove that the function $K^{\{m\}}(x, t)$ satisfies Eqs. (3.2), (3.3) and (3.5). For this purpose we first rewrite $R^{\{m\}}(x, t)$ as

$$
R^{\{m\}}(x, t)=\sum_{i=0}^{m-1} \frac{(x-a)^{i}}{i !} \frac{(t-a)^{i}}{i !}+\sum_{i=m}^{2 m-1}(-1)^{i-m} \frac{(x-a)^{2 m-i-1}}{(2 m-i-1) !} \frac{(t-a)^{i}}{i !} .
$$

For $j=0, \ldots, 2 m$, the $j^{t h}$-derivative of the function $R^{\{m\}}(x, t)$ with respect to $t$ is computed as

$\frac{\partial^{j} R^{\{m\}}(x, t)}{\partial t^{j}}= \begin{cases}\sum_{i=j}^{m-1} \frac{(x-a)^{i}}{i !} \frac{(t-a)^{i-j}}{(i-j) !}+\sum_{i=m}^{2 m-1}(-1)^{i-m} \frac{(x-a)^{2 m-i-1}}{(2 m-i-1) !} \frac{(t-a)^{i-j}}{(i-j) !}, & 0 \leq j<m, \\ \sum_{i=j}^{2 m-1}(-1)^{i-m} \frac{(x-a)^{2 m-i-1}}{(2 m-i-1) !} \frac{(t-a)^{i-j}}{(i-j) !}, & m \leq j<2 m, \\ 0, & j=2 m .\end{cases}$

Hence,

$$
\begin{gathered}
\left.\frac{\partial^{j} R^{\{m\}}(x, t)}{\partial t^{j}}\right|_{t=a}= \begin{cases}\frac{(x-a)^{j}}{j !}, & j=0, \ldots, m-1, \\
(-1)^{j-m} \frac{(x-a)^{2 m-j-1}}{(2 m-j-1) !}, & j=m, \ldots, 2 m-1,\end{cases} \\
\left.\frac{\partial^{j} R^{\{m\}}(x, t)}{\partial t^{j}}\right|_{t=x}= \begin{cases}\sum_{i=j}^{m-1} \frac{(x-a)^{2 i-j}}{i !(i-j) !}+\sum_{i=m}^{2 m-1}(-1)^{i-m} \frac{(x-a)^{2 m-j-1}}{(2 m-i-1) !(i-j) !}, & 0 \leq j<m, \\
\sum_{i=j}^{2 m-1}(-1)^{i-m} \frac{(x-a))^{2 m-j-1}}{(2 m-i-1) !(i-j) !}, & m \leq<2 m .\end{cases}
\end{gathered}
$$$$
\text { Also, we rewrite } L^{\{m\}}(x, t) \text { as }
$$$$
L^{\{m\}}(x, t)=R^{\{m\}}(t, x)=\sum_{i=0}^{m-1}\left[\frac{(x-a)^{i}}{i !}+(-1)^{m-i-1} \frac{(x-a)^{2 m-i-1}}{(2 m-i-1) !}\right] \frac{(t-a)^{i}}{i !} .
$$

Furthermore, for $j=0, \ldots, 2 m$, the $j^{t h}$-derivative of the function $L^{\{m\}}(x, t)$ with respect to $t$ is

$$
\frac{\partial^{j} L^{\{m\}}(x, t)}{\partial t^{j}}= \begin{cases}\sum_{i=j}^{m-1}\left(\frac{(x-a)^{i}}{i !}+(-1)^{m-i-1} \frac{(x-a)^{2 m-i-1}}{(2 m-i-1) !}\right) \frac{(t-a)^{i-j}}{(i-j) !}, & 0 \leq j<m, \\ 0, & m \leq j \leq 2 m .\end{cases}
$$


Therefore,

$$
\begin{gathered}
\left.\frac{\partial^{j} L^{\{m\}}(x, t)}{\partial t^{j}}\right|_{t=b}= \begin{cases}\sum_{i=j}^{m-1}\left(\frac{(x-a)^{i}}{i !}+(-1)^{m-i-1} \frac{(x-a)^{2 m-i-1}}{(2 m-i-1) !}\right) \frac{(b-a)^{i-j}}{(i-j) !}, & 0 \leq j<m, \\
0, & m \leq j<2 m\end{cases} \\
\left.\frac{\partial^{j} L^{\{m\}}(x, t)}{\partial t^{j}}\right|_{t=x}= \begin{cases}\sum_{i=j}^{m-1}\left(\frac{(x-a)^{2 i-j}}{i !(i-j) !}+(-1)^{m-i-1} \frac{(x-a)^{2 m-j-1}}{(2 m-i-1) !(i-j) !}\right), & 0 \leq j<m, \\
0, & m \leq j<2 m .\end{cases}
\end{gathered}
$$

Thus, by Eqs. (3.6) and (3.9), $\frac{\partial^{2 m} K^{\{m\}}(x, t)}{\partial t^{2 m}}=0$. Also, Eq. (3.7) implies

$$
\begin{aligned}
\left.\frac{\partial^{j} K^{\{m\}}(x, t)}{\partial t^{j}}\right|_{t=a} & -\left.(-1)^{m-j-1} \frac{\partial^{2 m-j-1} K^{\{m\}}(x, t)}{\partial t^{2 m-j-1}}\right|_{t=a} \\
& =\left.\frac{\partial^{j} R^{\{m\}}(x, t)}{\partial t^{j}}\right|_{t=a}-\left.(-1)^{m-j-1} \frac{\partial^{2 m-j-1} R^{\{m\}}(x, t)}{\partial t^{2 m-j-1}}\right|_{t=a} \\
& =\frac{(x-a)^{j}}{j !}-(-1)^{m-j-1}(-1)^{(2 m-j-1)-m} \frac{(x-a)^{2 m-(2 m-j-1)-1}}{(2 m-(2 m-j-1)-1) !} \\
& =\frac{(x-a)^{j}}{j !}-(-1)^{m-j-1}(-1)^{m-j-1} \frac{(x-a)^{j}}{j !}=0, \quad j=0, \ldots, m-1,
\end{aligned}
$$

and Eq. (3.10) implies

$$
\left.\frac{\partial^{j} K^{\{m\}}(x, t)}{\partial t^{j}}\right|_{t=b}=\left.\frac{\partial^{j} L^{\{m\}}(x, t)}{\partial t^{j}}\right|_{t=b}=0, j=m, \ldots, 2 m-1 .
$$

According to Eqs. (3.12) and (3.13), Eq. (3.3) is proved. Also, using Eqs. (3.8) and (3.11) we have

$$
\begin{aligned}
\left.\frac{\partial^{j} K^{\{m\}}(x, t)}{\partial t^{j}}\right|_{t}=x^{+} & =\left.\frac{\partial^{j} L^{\{m\}}(x, t)}{\partial t^{j}}\right|_{t=x} \\
& = \begin{cases}0, & m \leq j<2 m, \\
\sum_{i=j}^{m-1}\left(\frac{(x-a)^{2 i-j}}{i !(i-j) !}+(-1)^{m-i-1} \frac{(x-a)^{2 m-j-1}}{(2 m-i-1) !(i-j) !}\right), & 0 \leq j<m,\end{cases}
\end{aligned}
$$


TABLE 1. Explicit expressions of $R^{\{m\}}(x, t)$ for $m=1, \ldots, 5$

\begin{tabular}{|c|c|}
\hline$m$ & $R^{\{m\}}(x, t)$ \\
\hline \hline 1 & $1-a+t$ \\
\hline 2 & $1+(x-a)(t-a)+(x-a)(t-a)^{2} / 2-(t-a)^{3} / 6$ \\
\hline 3 & $1+(x-a)(t-a)+(x-a)^{2}(t-a)^{2} / 4+(x-a)^{2}(t-a)^{3} / 12$ \\
& $-(x-a)(t-a)^{4} / 24+(t-a)^{5} / 120$ \\
\hline 4 & $1+(x-a)(t-a)+(x-a)^{2}(t-a)^{2} / 4+(x-a)^{3}(t-a)^{3} / 36+(x-a)^{3}(t-a)^{4} / 144$ \\
& $-(x-a)^{2}(t-a)^{5} / 240+(x-a)(t-a)^{6} / 720-(t-a)^{7} / 5040$ \\
\hline 5 & $1+(x-a)(t-a)+(x-a)^{2}(t-a)^{2} / 4+(x-a)^{3}(t-a)^{3} / 36+(x-a)^{4}(t-a)^{4} / 576$ \\
& $+(x-a)^{4}(t-a)^{5} / 2880-(x-a)^{3}(t-a)^{6} / 4320+(x-a)^{2}(t-a)^{7} / 10080$ \\
& $-(x-a)(t-a)^{8} / 40320+(t-a)^{9} / 362880$ \\
\hline
\end{tabular}

So, Eqs. (3.14) and (3.15) imply that

$$
\begin{aligned}
& \left.\frac{\partial^{j} K^{\{m\}}(x, t)}{\partial t^{j}}\right|_{t=x^{+}}-\left.\frac{\partial^{j} K^{\{m\}}(x, t)}{\partial t^{j}}\right|_{t=x^{-}} \\
& = \begin{cases}\sum_{i=j}^{m-1} \frac{(-1)^{m+i-1}(x-a)^{2 m-j-1}}{(2 m-i-1-1) !(i-j) !}+\sum_{i=m}^{2 m-1} \frac{(-1)^{m+i-1}(x-a)^{2 m-j-1}}{(2 m-i-1) !(i-j) !}, & 0 \leq j<m, \\
(x-a)^{2 m-j-1} \sum_{i=j}^{2 m-1} \frac{(-1)^{m+i-1}}{(2 m-i-1) !(i-j) !}, & m \leq j<2 m-1, \\
(-1)^{m}, & j=2 m-1 .\end{cases} \\
& = \begin{cases}(-1)^{m-1}(x-a)^{2 m-j-1} \sum_{i=j}^{2 m-1} \frac{(-1)^{i}}{(2 m-i-1) !(i-j) !}, & 0 \leq j \leq 2 m-2, \\
(-1)^{m}, & j=2 m-1 .\end{cases} \\
& = \begin{cases}\frac{(-1)^{m+j-1}(x-a)^{2 m-j-1}}{(-1)^{m},} \sum_{i=0}^{2 m-j-1) !}(-1)^{i}\left(\begin{array}{cl}
2 m-j-1 \\
i
\end{array}\right), & 0 \leq j \leq 2 m-2, \\
& j=2 m-1 .\end{cases} \\
& = \begin{cases}(-1)^{m+j-1}(x-a)^{2 m-j-1}(1-1)^{2 m-j-1}, & 0 \leq j \leq 2 m-2, \\
(-1)^{m}, & j=2 m-1 .\end{cases} \\
& = \begin{cases}0, & 0 \leq j \leq 2 m-2, \\
(-1)^{m}, & j=2 m-1 .\end{cases}
\end{aligned}
$$

Explicit expressions of the reproducing kernel functions in $W_{2}^{m}[a, b]$ for $1 \leq m \leq 5$ are shown in Table 1.

\subsection{Reproducing kernel of the space $W_{2, a}^{n+1}[a, b]$}

Suppose that function $K_{a}^{\{n+1\}}(x, t) \in W_{2, a}^{n+1}[a, b]$ satisfies the following generalized differential equations

$$
\begin{cases}(-1)^{n+1} \frac{\partial^{2 n+2} K_{a}^{\{n+1\}}(x, t)}{\partial t^{2 n+2}}=\delta(t-x), & \\ \left.\frac{\partial^{n+1} K_{a}^{\{n+1\}}(x, t)}{\partial t^{n+1}}\right|_{t=a}=\left.\frac{\partial^{n} K^{\{n+1\}}(x, t)}{\partial t^{n}}\right|_{t=a}, & 0 \leq j \leq n, \\ \left.\frac{\partial^{j} K_{a}^{\{n+1\}}(x, t)}{\partial t^{j}}\right|_{t=a}=0, & 0 \leq j \leq n, \\ \left.\frac{\partial^{j} K_{a}^{\{n+1\}}(x, t)}{\partial t^{j}}\right|_{t=b}=0, & n<j<2 n,\end{cases}
$$


where $\delta$ is the Dirac delta function. Similar to Theorem 1, we have the following theorem.

Theorem 3. Under the assumptions of Eq. (3.16), Hilbert space $W_{2, a}^{n+1}[a, b]$ is a reproducing kernel Hilbert space with the reproducing kernel function $K_{a}^{\{n+1\}}(x,$.$) ,$ namely for each $u \in W_{2, a}^{n+1}[a, b]$ and any fixed $x \in[a, b]$, it follows that

$$
\left\langle u(.), K_{a}^{\{n+1\}}(x, .)\right\rangle_{n, a}=u(x) .
$$

While $x \neq t$, function $K_{a}^{\{n+1\}}(x, t)$ is the solution of the following linear homogeneous differential equation of order $2 n+2$,

$$
(-1)^{n+1} \frac{\partial^{2 n+2} K_{a}^{\{n+1\}}(x, t)}{\partial t^{2 n+2}}=0
$$

with the boundary conditions:

$$
\begin{cases}\left.\frac{\partial^{n+1} K_{a}^{\{n+1\}}(x, t)}{\partial t^{n+1}}\right|_{t=a}=\left.\frac{\partial^{n} K^{\{n+1\}}(x, t)}{\partial t^{n}}\right|_{t=a}, & 0 \leq j \leq n, \\ \left.\frac{\partial^{j} K_{a}^{\{n+1\}}(x, t)}{\partial t^{j}}\right|_{t=a}=0, & 0 \leq j \leq n, \\ \left.\frac{\partial^{j} K_{a}^{\{n+1\}}(x, t)}{\partial t^{j}}\right|_{t=b}=0, & n<j<2 n .\end{cases}
$$

We know that Eq. (3.17) has characteristic equation $\lambda^{2 n+2}=0$, and the eigenvalue $\lambda=0$ is a root with multiplicity $2 n+2$. Hence, the general solution of the system (3.16) is

$$
K_{a}^{\{n+1\}}(x, t)= \begin{cases}\sum_{i=1}^{2 n+2} \alpha_{i}(x)(t-a)^{i-1}, & t \leq x, \\ \sum_{i=1}^{2 n+1} \beta_{i}(x)(t-a)^{i-1}, & t>x .\end{cases}
$$

Now, we are ready to calculate the coefficients $\alpha_{i}(x)$ and $\beta_{i}(x), i=1, \ldots, 2 n+2$. Since

$$
(-1)^{n+1} \frac{\partial^{2 n+2} K_{a}^{\{n+1\}}(x, t)}{\partial t^{2 n+2}}=\delta(t-x),
$$

we have

$$
\begin{cases}\left.\frac{\partial^{j} K_{a}^{\{n+1\}}(x, t)}{\partial t^{j}}\right|_{t=x^{-}} ^{t=x^{+}}=0, & j=0, \ldots, 2 n, \\ \left.\frac{\partial^{j} K_{a}^{\{n+1\}}(x, t)}{\partial t^{j}}\right|_{t=x^{-}} ^{t=x^{+}}=(-1)^{n+1}, & j=2 n+1 .\end{cases}
$$

Therefore, using Eqs. (3.16) and (3.20), we have a system of $4 n+4$ equations with $4 n+4$ unknowns $\left(\alpha_{i}(x)\right.$ and $\left.\beta_{i}(x), i=1, \ldots, 2 n+2\right)$. By solving this system the unknown coefficients of Eq. (3.19) are uniquely obtained. Solving the above system needs high CPU time.

Now, we present the following formula for calculating the reproducing kernel of $W_{2, a}^{n+1}[a, b]$. 
Theorem 4. Let

$$
K_{a}^{\{n+1\}}(x, t)= \begin{cases}R_{a}^{\{n+1\}}(x, t), & t \leq x, \\ L_{a}^{\{n+1\}}(x, t), & t>x,\end{cases}
$$

be the reproducing kernel function of the reproducing kernel Hilbert space $W_{2, a}^{n+1}[a, b]$. Then, $R_{a}^{\{n+1\}}(x, t)$ is given by the following formula.

$$
R_{a}^{\{n+1\}}(x, t)=\frac{(x-a)^{n}(t-a)^{n}}{n ! n !}+\sum_{i=n+1}^{2 n+1}\left((-1)^{i-n-1} \frac{(x-a)^{2 n-i+1}(t-a)^{i}}{(2 n-i+1) ! i !}\right),
$$

and $L_{a}^{\{n+1\}}(x, t)=R_{a}^{\{n+1\}}(t, x)$.

Proof of Theorem 4. It is enough to prove that the function $K_{a}^{\{n+1\}}(x, t)$ satisfies in Eqs. (3.17), (3.18) and (3.20). For $j=0, \ldots, 2 n+2$, the $j^{\text {th }}$-derivative of the function $R_{a}^{\{n+1\}}(x, t)$ with respect to $t$ is computed as follows:

$$
\frac{\partial^{j} R_{a}^{\{n+1\}}(x, t)}{\partial t^{j}}= \begin{cases}\frac{(x-a)^{n}}{n !} \frac{(t-a)^{n-j}}{(n-j) !}+ \\ \sum_{i=n+1}^{2 n+1}(-1)^{i-n-1} \frac{(x-a)^{2 n-i+1}}{(2 n-i+1) !} \frac{(t-a)^{i-j}}{(i-j) !}, & 0 \leq j<n+1, \\ \sum_{i=j}^{2 n+1}(-1)^{i-n-1} \frac{(x-a)^{2 n-i+1}}{(2 n-i+1) !} \frac{\left.(t-a)^{i}\right) !}{(i-j) !}, & n<j<2 n+2, \\ 0, & j=2 n+2 .\end{cases}
$$

Then,

$$
\begin{gathered}
\left.\frac{\partial^{j} R_{a}^{\{n+1\}}(x, t)}{\partial t^{j}}\right|_{t=a}= \begin{cases}\frac{(x-a)^{j}}{j !}, & j=n, \\
(-1)^{j-n-1} \frac{(x-a)^{2 n-j+1}}{(2 n-j+1) !}, & n<j<2 n,\end{cases} \\
\left.\frac{\partial^{j} R_{a}^{\{n+1\}}(x, t)}{\partial t^{j}}\right|_{t=x}= \begin{cases}\frac{(x-a)^{2 n-j}}{n !(n-j) !}+ & \begin{array}{ll}
\sum_{i=n+1}^{2 n+1}(-1)^{i-n-1} \frac{(x-a)^{2 n-j+1}}{(2 n-i)^{2 n ! !}(i-j) !}, & 0 \leq j \leq n, \\
\sum_{i=j}^{2 n+1}(-1)^{i-n-1} \frac{(x-a)^{2 n-j+1+1}}{(2 n-i+1) !(i-j) !}, & n<j<2 n .
\end{array}\end{cases}
\end{gathered}
$$

For $L_{a}^{\{n+1\}}(x, t)$ we have

$$
L_{a}^{\{n+1\}}(x, t)=R_{a}^{\{n+1\}}(t, x)=\frac{(x-a)^{n}}{n !} \frac{(t-a)^{n}}{n !}+\sum_{i=0}^{n}(-1)^{n-i} \frac{(x-a)^{2 n-i+1}}{(2 n-i+1) !} \frac{(t-a)^{i}}{i !} .
$$

Furthermore, for $j=0, \ldots, 2 n+2$, the $j^{t h}$-derivative of the function $L_{a}^{\{n+1\}}(x, t)$ with respect to $t$ is computed as follows:

$$
\frac{\partial^{j} L_{a}^{\{n+1\}}(x, t)}{\partial t^{j}}= \begin{cases}\frac{(x-a)^{n}}{n !} \frac{(t-a)^{n-j}}{(n-j) !}+ & \\ \sum_{i=j}^{n}(-1)^{n-i} \frac{(x-a)^{2 n-i+1}}{(2 n-i+1) !} \frac{(t-a)^{i-j}}{(i-j) !}, & 0 \leq j<n+1, \\ 0, & n<j \leq 2 n+2 .\end{cases}
$$

Therefore,

$$
\left.\frac{\partial^{j} L_{a}^{\{n+1\}}(x, t)}{\partial t^{j}}\right|_{t=b}= \begin{cases}\frac{(x-a)^{n}}{n !} \frac{(b-a)^{n-j}}{(n-j) !}+ \\ \sum_{i=j}^{n !}(-1)^{n-i} \frac{(x-a)^{2 n-i+1}}{(2 n-i+1) !} \frac{(b-a)^{i-j}}{(i-j) !}, & 0 \leq j<n+1, \\ 0, & n<j \leq 2 n+2,\end{cases}
$$




$$
\left.\frac{\partial^{j} L_{a}^{\{n+1\}}(x, t)}{\partial t^{j}}\right|_{t=x}= \begin{cases}\frac{(x-a)^{2 n-j}}{n !(n-j) !}+ & \\ \sum_{i=j}^{n}(-1)^{n-i} \frac{(x-a)^{2 n-j+1}}{(2 n-i+1) !(i-j) !}, & 0 \leq j<n+1, \\ 0, & n<j \leq 2 n+2 .\end{cases}
$$

Thus, Eqs. (3.21) and (3.24), it is clear that $\frac{\partial^{2 n+2} K_{a}^{\{n+1\}}(x, t)}{\partial t^{2 n+2}}=0$. Also, Eq. (3.22) implies that

$$
\begin{gathered}
\left.\frac{\partial^{j} K_{a}^{\{n+1\}}(x, t)}{\partial t^{j}}\right|_{t=a}=\left.\frac{\partial^{j} L_{a}^{\{n+1\}}(x, t)}{\partial t^{j}}\right|_{t=a}=0, \quad j=0, \ldots, n-1, \\
\left.\frac{\partial^{n+1} K_{a}^{\{n+1\}}(x, t)}{\partial t^{n+1}}\right|_{t=a}-\left.\frac{\partial^{n} K_{a}^{\{n+1\}}(x, t)}{\partial t^{n}}\right|_{t=a}= \\
\left.\frac{\partial^{n+1} L_{a}^{\{n+1\}}(x, t)}{\partial t^{n+1}}\right|_{t=a}-\left.\frac{\partial^{n} L_{a}^{\{n+1\}}(x, t)}{\partial t^{n}}\right|_{t=a}=\frac{(x-a)^{n}}{n !}-\frac{(x-a)^{n}}{n !}=0,
\end{gathered}
$$

and Eq. (3.25) implies that

$$
\left.\frac{\partial^{j} K_{a}^{\{n+1\}}(x, t)}{\partial t^{j}}\right|_{t=b}=\left.\frac{\partial^{j} L_{a}^{\{n+1\}}(x, t)}{\partial t^{j}}\right|_{t=b}=0, n<j<2 n .
$$

According to Eqs. (3.27) and (3.28), Eq. (3.18) is proved. Also, using Eqs. (3.23) and (3.26) we have

$$
\begin{aligned}
\left.\frac{\partial^{j} K_{a}^{\{n+1\}}(x, t)}{\partial t^{j}}\right|_{t=x^{+}} & =\left.\frac{\partial^{j} L_{a}^{\{n+1\}}(x, t)}{\partial t^{j}}\right|_{t=x} \\
& = \begin{cases}0, & n<j<2 n, \\
\frac{(x-a)^{2 n-j}}{n !(n-j) !}+\sum_{i=j}^{n}(-1)^{n-i} \frac{(x-a)^{2 n-j+1}}{(2 n-i+1) !(i-j) !}, & 0 \leq j \leq n,\end{cases} \\
\left.\frac{\partial^{j} K_{a}^{\{n+1\}}(x, t)}{\partial t^{j}}\right|_{t=x^{-}} & =\left.\frac{\partial^{j} R_{a}^{\{n+1\}}(x, t)}{\partial t^{j}}\right|_{t=x} \\
& = \begin{cases}\sum_{i=j}^{2 n+1}(-1)^{i-n-1} \frac{(x-a)^{2 n-j+1}}{(2 n-i+1) !(i-j) !}, & n<j<2 n, \\
\frac{(x-a)^{2 n-j}}{n !(n-j) !}+\sum_{i=n+1}^{2 n+1}(-1)^{i-n-1} \frac{(x-a)^{2 n-j+1}}{(2 n-i+1) !(i-j) !}, & 0 \leq j \leq n .\end{cases}
\end{aligned}
$$

So, Eqs. (3.29) and (3.30) imply that

$$
\begin{aligned}
\left.\frac{\partial^{j} K_{a}^{\{n+1\}}(x, t)}{\partial t^{j}}\right|_{t} & x^{+}-\left.\frac{\partial^{j} K_{a}^{\{n+1\}}(x, t)}{\partial t^{j}}\right|_{t=x^{-}} \\
& = \begin{cases}\sum_{i=j}^{n} \frac{(-1)^{n+i}(x-a)^{2 n-j+1}}{(2 n-i+1)(i-j) !}+\sum_{i=n+1}^{2 n+1} \frac{(-1)^{n+i}(x-a)^{2 n-j+1}}{(2 n-i+1) !(i-j) !}, & 0 \leq j<n+1, \\
(x-a)^{2 n-j+1} \sum_{i=j}^{2 n+1} \frac{(-1)^{n+i}}{(2 n-i+1) !(i-j) !}, & n<j<2 n+1, \\
(-1)^{n+1}, & j=2 n+1 .\end{cases} \\
& = \begin{cases}(-1)^{n}(x-a)^{2 n-j+1} \sum_{i=j}^{2 n+1} \frac{(-1)^{i}}{(2 n-i+1) !(i-j) !}, & 0 \leq j \leq 2 n, \\
(-1)^{n+1}, & j=2 n+1 .\end{cases} \\
& = \begin{cases}\frac{(-1)^{n+j}(x-a)^{2 n-j+1}(2 n-j+1) !}{(-1)^{n+1},} \sum_{i=0}^{2 n-j+1}(-1)^{i}(2 n-j+1), & 0 \leq j \leq 2 n, \\
i & j=2 n+1 .\end{cases} \\
& = \begin{cases}(-1)^{n+j}(x-a)^{2 n-j+1}(1-1)^{2 n-j+1}, & 0 \leq j \leq 2 n, \\
(-1)^{n+1}, & j=2 n+1 . \\
0, & 0 \leq j \leq 2 n, \\
(-1)^{n+1}, \quad j=2 n+1 .\end{cases}
\end{aligned}
$$


TABLE 2. Explicit expressions of $R_{a}^{\{n+1\}}(x, t)$ for $n=1, \ldots, 4$

\begin{tabular}{|c|c|}
\hline$n$ & $R_{a}^{\{n+1\}}(x, t)$ \\
\hline \hline 1 & $(x-a)(t-a)+(x-a)(t-a)^{2} / 2-(t-a)^{3} / 6$ \\
\hline 2 & $(x-a)^{2}(t-a)^{2} / 4+(x-a)^{2}(t-a)^{3} / 12-(x-a)(t-a)^{4} / 24+(t-a)^{5} / 120$ \\
\hline 3 & $(x-a)^{3}(t-a)^{3} / 36+(x-a)^{3}(t-a)^{4} / 144-(x-a)^{2}(t-a)^{5} / 240$ \\
& $+(x-a)(t-a)^{6} / 720-(t-a)^{7} / 5040$ \\
\hline 4 & $(x-a)^{4}(t-a)^{4} / 576+(x-a)^{4}(t-a)^{5} / 2880-(x-a)^{3}(t-a)^{6} / 4320$ \\
& $+(x-a)^{2}(t-a)^{7} / 10080-(x-a)(t-a)^{8} / 40320+(t-a)^{9} / 362880$ \\
\hline
\end{tabular}

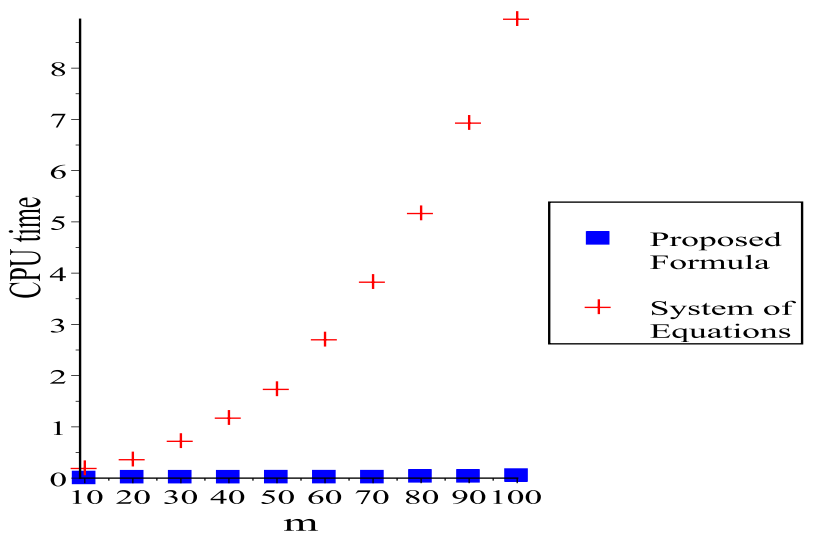

FIGURE 1. Comparing CPU time to compute the reproducing kernel for space $W_{2}^{m}[a, b]$

Explicit expressions of reproducing kernel functions in $W_{2, a}^{n+1}[a, b]$ for $n=1, \ldots, 4$, are shown in Table 2.

Remark 1. For some values of $m$ (or $n$ ), we have calculated the reproducing kernel, using two methods: the proposed explicit formula, and also by solving the system of $4 m$ (or $4 n+4)$ equations in $4 m$ (or $4 n+4)$ unknowns. Figures 1 and 2 , represent the advantage of the explicit formulas.

\section{CONCLUSiOnS}

In this paper we examined some reproducing kernel spaces and we obtained explicit formulas for the reproducing kernel of these spaces. Usually finding the reproducing kernel for solving large systems of differential equations is required (especially for high-order differential equations). For an $n$ th-order differential equation, we must solve a system of $4(n+1)$ equations in $4(n+1)$ unknowns which is very time-consuming and therefore an explicit formula for these reproducing kernels is 


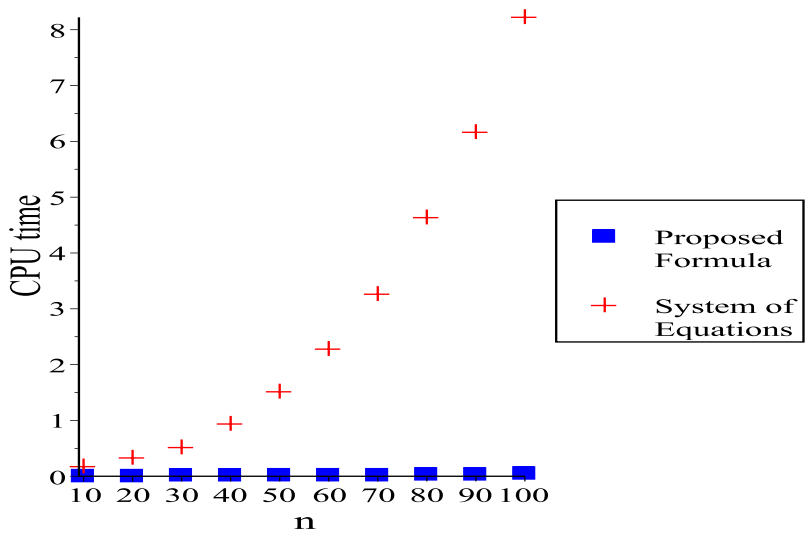

FIGURE 2. Comparing CPU time to compute the reproducing kernel for space $W_{2}^{n+1}[a, b]$

very useful (see Figures 1 and 2).

\section{ACKNOWLEDGEMENT}

The authors thank the anonymous referees of this paper for their careful reading, constructive comments and suggestions.

\section{REFERENCES}

[1] N. Aronszajn, "Theory of reproducing kernel," Trans. Amer. Math. Soc., vol. 68, pp. 337-404, 1950, doi: 10.1090/S0002-9947-1950-0051437-7.

[2] Z. Chen and W. Jiang, "The exact solution of a class of volterra integral equation with weakly singular kernel," Appl. Math. Comput., vol. 217, pp. 7515-7519, 2011.

[3] Z. Chen and Y. Zhou, "A new method for solving hilbert type singular integral equations," Appl. Math. Comput., vol. 218, pp. 406-412, 2011.

[4] M. Cui and Y. Lin, Nonlinear Numerical Analysis in Reproducing Kernel Hilbert Space. Nova Science Publisher, 2009.

[5] H. Du and M. Cui, "Representation of the exact solution and stability analysis on the fredholm integral equation of the frist kind in a reproducing kernel space," Appl. Math. Comput., vol. 182, pp. 1608-1614, 2006, doi: 10.1016/j.amc.2006.05.049.

[6] H. Du and M. Cui, "Approximate solution of the fredholm integral equation of the frist kind in a reproducing kernel space," Appl. Math. Letters, vol. 21, pp. 617-623, 2008, doi: 10.1016/j.aml.2007.07.014.

[7] F. Geng and M. Cui, "Solving a nonlinear system of second order boundary value problems," $J$. Math. Anal. Appl., vol. 327, pp. 1167-1181, 2007, doi: 10.1016/j.jmaa.2006.05.011.

[8] F. Geng and M. Cui, "Solving singular nonlinear second-order periodic boundary value problems in the reproducing kernel space," Appl. Math. Comput., vol. 192, pp. 389-398, 2007, doi: 10.1016/j.amc.2007.03.016. 
[9] F. Geng and M. Cui, "Reproducing kernel method for solving tenth-order boundary value problems," J. Appl. Math. Informatics, vol. 28, pp. 818-821, 2010.

[10] F. Geng and M. Cui, "Homotopy perturbation reproducing kernel method for nonlinear systems of second order boundary value problems," J. Comput. Appl. Math., vol. 235, pp. 2405-2411, 2011.

[11] F. Geng and M. Cui, "A reproducing kernel method for solving nonlocal fractional boundary value problems," Appl. Math. Letters, vol. 25, pp. 818-823, 2012, doi: 10.1016/j.aml.2011.10.025.

[12] F. Geng and S. Qian, "Reproducing kernel method for singularly perturbed turning point problems having twin boundary layers," Appl. Math. Letters, vol. 26, pp. 998-1004, 2013, doi: 10.1016/j.aml.2013.05.006.

[13] F. Geng, S. Qian, and S. Li, "A numerical method for singularly perturbed turning point problems with an interior layer," J. Comput. Appl. Math., vol. 255, pp. 97-105, 2013, doi: 10.1016/j.cam.2013.04.040.

[14] W. Jiang and M. Cui, "The exact solution and stability analysis for integral equation of third or frist kind with singular kernel," Appl. Math. Comput., vol. 202, pp. 666-674, 2008, doi: 10.1016/j.amc.2008.03.005.

[15] W. Jiang, M. Cui, and Y. Lin, "Anti-periodic solutions for rayleigh-type equations via the reproducing kernel hilbert space method," Commun Nonlinear Sci Numer Simulat, vol. 15, pp. 1754-1758, 2010, doi: 10.1016/j.cnsns.2009.07.022.

[16] R. Ketabchi, R. Mokhtari, and E. Babolian, "Some error estimates for solving volterra integral equations by using the reproducing kernel method," J. Comput. Appl. Math., vol. 273, pp. 245250, 2015, doi: 10.1016/j.cam.2014.06.016.

[17] X. Lin and B. Wu, "Error estimation for the reproducing kernel method to solve linear boundary value problems," J. Comput. Appl. Math., vol. 243, pp. 10-15, 2013, doi: 10.1016/j.cam.2012.11.002.

[18] M. Mohammadi and R. Mokhtari, "Solving the generalized regularized long wave equation on the basis of a reproducing kernel space," J. Comput. Appl. Math., vol. 235, pp. 4003-4014, 2011, doi: 10.1016/j.cam.2011.02.012.

[19] W. Wang, M. Cui, and B. Han, "A new method for solving a class of singular twopoint boundary value problems," Appl. Math. Comput., vol. 206, pp. 721-727, 2008, doi: 10.1016/j.amc.2008.09.019.

[20] Y. Wang, L. Su, X. Cao, and X. Li, "Using reproducing kernel for solving a class of singularly perturbed problems," Comput. Math. Appl., vol. 61, pp. 421-430, 2011, doi: 10.1016/j.camwa.2010.11.019.

[21] B. Wu and X. Li, "Iterative reproducing kernel method for nonlinear oscillator with discontinuity," Appl. Math. Letters, vol. 23, pp. 1301-1304, 2010, doi: 10.1016/j.aml.2010.06.018.

[22] L. Yang, J. Shen, and Y. Wang, "The reproducing kernel method for solving the system of linear volterra integral equations with variable coefficients," J. Comput. Appl. Math., vol. 236, pp. 2398 2405, 2012, doi: 10.1016/j.cam.2011.11.026.

[23] H. Yao and Y. Lin, "Solving singular boundary-value problems of higher even-order," J. Comput. Appl. Math., vol. 223, pp. 703-713, 2009, doi: 10.1016/j.cam.2008.02.010.

[24] W. Yulan, T. Chaolu, and P. Jing, "New algorithm for second-order boundary value problems of integro-differential equation," J. Comput. Appl. Math., vol. 229, pp. 1-6, 2009, doi: 10.1016/j.cam.2008.10.007.

[25] Y. L. Z. Chen, "The exact solution of a class of linear integral equation with weakly singular kernel," J. Math. Anal. Appl., vol. 344, pp. 726-734, 2008, doi: 10.1016/j.amc.2011.02.059. 
Authors' addresses

\section{E. Moradi}

Kharazmi University, Department of Mathematics, Faculty of Mathematical Sciences and Computer, 50 Taleghani Avenue, 1561836314 Tehran, Iran

E-mail address: std_e.moradi@khu.ac.ir

\section{E. Babolian}

Kharazmi University, Department of Mathematics, Faculty of Mathematical Sciences and Computer, 50 Taleghani Avenue, 1561836314 Tehran, Iran

E-mail address: babolian@khu.ac.ir

\section{S. Javadi}

Kharazmi University, Department of Mathematics, Faculty of Mathematical Sciences and Computer, 50 Taleghani Avenue, 1561836314 Tehran, Iran

E-mail address: shahnam.javadi@gmail.com 